ANNALES

POLONICI MATHEMATICI

XIV (1963)

\title{
Sur une équation diophantienne considérée par Goormaghtigh
}

\author{
par Chr. KaranicolofF (Sofia)
}

Pour l'équation diophantienne

(1)

$$
\begin{aligned}
N=1+x+x^{2}+\ldots+x^{m}=1+y+y^{2}+\ldots+y^{n} & \\
& (m>n>1 ; y>x>1)
\end{aligned}
$$

R. Goormaghtigh [1] a montré que, si $N<10^{6}$, elle n'a d'autres solutions en nombres naturels $[N, m, n, x, y]$ que les deux solutions suivantes

$$
[31,4,2,2,5], \quad[8191,12,2,2,90] \text {. }
$$

Récemment $H$. J. Kanold [2] a démontré que si les inconnues $x, y$ sont fixées, l'équation (1) n'aura qu'un nombre fini de solutions $[m, n]$, ce nombre pouvant être égal à zéro.

A. Schinzel et A. Mazkowski [3] viennent de publier certains cas particuliers de la solution de (1) et ils en ont donné quelques propriétés intéréssantes.

A notre tour, nous allons démontrer (théorème II) que dans le cas assez général

$$
m=2 n \quad(n>1)
$$

l'équation (1) ne possède qu'une solution unique et que cette solution est précisément celle qui a été trouvée par Goormaghtigh, soit $[31,4,2,2,5]$.

D'abord, nous établirons le théorème suivant.

THéonغ̀me I. Si l'équation (1) admet - $m, n$ étant fixés - la solution $\left[N_{1}, p, q\right]$, le premier de ces nombres $\left(N_{1}\right)$ satisfait au système suivant:

$$
\begin{aligned}
& {\left[N^{1 / n}\right]\left(N-\left[N^{1 / n}\right]^{n}\right)=N-1,} \\
& {\left[N^{1 / m}\right]\left(N-\left[N^{1 / m}\right]^{m}\right)=N-1,} \\
& {\left[N^{1 / m}\right]>1, \quad\left[N^{1 / n}\right]>2 .}
\end{aligned}
$$

En effet, comme $\left[N_{1}, p, q\right]$ est par hypothèse une solution de (1), nous aurons

$$
N_{1}=p^{m}+p^{m-1}+\ldots+p+1
$$


et

$$
N_{1}=q^{n}+q^{n-1}+\ldots+q+1 .
$$

Partant de (6) nous trouvons successivement

soit

$$
\begin{aligned}
N_{1}^{1 / m} & =\left(p^{m}+p^{m-1}+\ldots+p+1\right)^{1 / m} \\
& =p\left(1+\frac{1}{p}+\frac{1}{p^{2}}+\ldots+\frac{1}{p^{m}}\right)^{1 / m},
\end{aligned}
$$

$$
N_{1}^{1 / m}=p+\sigma
$$

où

c'est-à-dire

$$
\sigma=p \sum_{k=1}^{\infty}\left(\begin{array}{c}
1 / m \\
k
\end{array}\right)\left(\frac{1}{p}+\frac{1}{p^{2}}+\ldots+\frac{1}{p^{m}}\right)^{k}
$$

(9) $\quad \sigma=\frac{1+p^{-1}+p^{-2}+\ldots+p^{-(m-1)}}{m}-\frac{(m-1) p\left(p^{-1}+p^{-2}+\ldots+p^{-m}\right)^{2}}{2 m^{2}}+\ldots$

Il est facile de voir que la série alternante au second membre de (9) satisfait aux conditions du critère de convergence de Leibniz et que son premier membre atteint sa valeur maxima pour $p=2$, lorsque $p$ varie dans l'intervalle $[2,+\infty)$ et par conséquent

$$
0<\sigma<\frac{1+2^{-1}+2^{-2}+\ldots+2^{-(m-1)}}{m}=\frac{2^{m}-1}{m 2^{m-1}} .
$$

Mais, comme $m>n>1$, done $m \geqslant 3$, de (10) découlent les inégalités

$$
0<\sigma<1 \text {. }
$$

De celles-ci, ainsi que directement de (8), nous déduisons que

$$
\left[N_{1}^{1 / m}\right]=[p+\sigma]=p \text {. }
$$

D'autre part, en partant non pas de (6), mais de (7), nous pouvons démontrer que

$$
\left[N_{1}^{1 / n}\right]=\left[q+\sigma_{1}\right]=q \quad\left(0<\sigma_{1}<1\right) .
$$

Si maintenant nous remplaçons dans l'identité

$$
p\left[\left(p^{m}+p^{m-1}+\ldots+p+1\right)-p^{m}\right]=\left(p^{m}+p^{m-1}+\ldots+p+1\right)-1
$$

l'expression $\left(p^{m}+p^{m-1}+\ldots+p+1\right)$ par celle qui lui est égale d'après (6), nous obtiendrons

$$
p\left(N_{1}-p^{m}\right)=N_{1}-1
$$


et en éliminant $p$ de cette égalité et de (11), nous trouvons l'égalité

$$
\left[N_{1}^{1 / m}\right]\left(N_{1}-\left[N_{1}^{1 / m}\right]^{m}\right)=N_{1}-1
$$

qui montre que le nombre $N_{1}$ satisfait à l'équation (4).

De manière analogue, en partant de l'identité

$$
q\left[\left(q^{n}+q^{n-1}+\ldots+q+1\right)-q^{n}\right]=\left(q^{n}+q^{n-1}+\ldots+q+1\right)-1
$$

et des égalités (7) et (12), nous pouvons prouver que le même nombre $N_{1}$ satisfait à l'équation (3) du système (A).

Enfin, pour démontrer que le nombre $N_{1}$ satisfait aussi aux inégalités (5), il suffit de remarquer que nous disposons des égalités (11) et (12) et de la condition

$$
q>p \geqslant 2
$$

De ce fait le théorème I est prouvé. Il en découle directement le corollaire suivant.

Corollame. Dans le cas où, $m, n$ étant fixés, le système (A) n'admet aucune solution en nombres naturels par rapport à l'inconnue $N$ l'équation (1) aussi ne possèdera point de solution $[N, x, y]$.

Par contre, il est facile de voir qu'à chaque solution $N_{1}$ du système (A) correspond une solution $\left[N_{0}, x_{0}, y_{0}\right]$ de l'équation (1), laquelle solution est définie par les égalités

$$
N_{0}=N_{1}, \quad x_{0}=\left[N_{1}^{1 / m}\right], \quad y_{0}=\left[N_{1}^{1 / n}\right] .
$$

Théorìme II. Dans le cas $m=2 n$ l'équation (1) n'admet que la solution $[31,4,2,2,5]$.

LEMME. Si $m=2 n$ ( $n \geqslant 2)$, l'équation (1) $n$ 'a de solution $[N, m, n, x, y]$ $\neq[31,4,2,2,5]$ que dans le cas où l'équation

$$
\left(p^{2}+k\right)\left[p^{2 n}+p^{2 n-1}+\ldots+p+1-\left(p^{2}+k\right)^{n}\right]=p^{2 n}+p^{2 n-1}+\ldots+p^{2}+p
$$

a des solutions $[p, k]$ en nombres entiers, satisfaisant aux inégalités

$$
0<k<\frac{p}{n} \quad(p \geqslant 2) \text {. }
$$

Démonstration. En posant dans l'équation (1): $x=p, y=p^{2}+k$, nous trouvons évidemment $p \geqslant 2, k>0$ et comme

$$
\begin{aligned}
& \left(p^{2}+k-1\right)\left[p^{2 n}+p^{2 n-1}+\ldots+1-\left(p^{2}+k\right)^{n}-\left(p^{2}+k\right)^{n-1}-\ldots-1\right] \\
& \quad=\left(p^{2}+k\right)\left[p^{2 n}+p^{2 n-1}+\ldots+p+1-\left(p^{2}+k\right)^{n}\right]-p^{2 n}-p^{2 n-1}-\ldots-p
\end{aligned}
$$

les nombres entiers $p, k$ satisfont à l'équation (15). 
Alors,

Pour démontrer que $k<p / n$ supposons au contraire que $k \geqslant p / n$.

d'où

$$
\begin{gathered}
\left(p^{2}+k\right)^{n} \geqslant\left(p^{2}+\frac{p}{n}\right)^{n} \geqslant p^{2 n}+p^{2 n-1}+\frac{n-1}{2 n} p^{2 n-2} \\
\left(p^{2}+k\right)^{i} \geqslant p^{2 i} \quad(i=0,1, \ldots, n-1)
\end{gathered}
$$

$$
\begin{aligned}
\left(p^{2}+k\right)^{n}+\left(p^{2}+k\right)^{n-1} & +\ldots+1-p^{2 n}-p^{2 n-1}-\ldots-1 \\
\geqslant & \frac{n-1}{2 n} p^{2 n-2}-p^{2 n-3}-p^{2 n-5}-\ldots-p \\
& =\frac{n-1}{2 n} p^{2 n-2}-p \frac{p^{2 n-2}-1}{p^{2}-1}>p^{2 n-2}\left(\frac{n-1}{2 n}-\frac{p}{p^{2}-1}\right) .
\end{aligned}
$$

Si $n>2$ et $p>2$ (sauf le cas: $n=3, p=3$ ) ou $n=2$ et $p>4$ on a

d'où

$$
\frac{n-1}{2 n}-\frac{p}{p^{2}-1}>0
$$

$$
\left(p^{2}+k\right)^{n}+\left(p^{2}+k\right)_{1}^{n-1}+\ldots+1-p^{2 n}-p^{2 n-1}-\ldots-1>0 .
$$

Dans les cas: 1) $n=2, p=3$; 2) $n=3, p=3$; 3) $n=2, p=4$ (et $k \geqslant p / n$ ) l'inégalité (17) se vérifie directement.

Enfin, si $p=2, n \geqslant 3$ et $k \geqslant p / n$ on a

$$
\begin{aligned}
\left(p^{2}+k\right)^{n}+\ldots+1-p^{2 n}-\ldots-1 \geqslant\left(4+\frac{2}{n}\right)^{n}+\ldots+1-2^{2 n}-\ldots-1 \\
\quad=\frac{n}{3 n+2}\left[\left(4+\frac{2}{n}\right)^{n+1}-1\right]-2^{2 n+1}+1 \\
\quad>\frac{n}{3 n+2}\left[\left(4+\frac{2}{n}\right)^{n+1}-2^{2 n+1}\left(3+\frac{2}{n}\right)\right] \geqslant 4^{n}\left[\left(1+\frac{1}{2 n}\right)^{n+1}-\frac{3}{2}-\frac{1}{n}\right] \\
\geqslant 4^{n}\left[1+\frac{n+1}{2 n}+\frac{(n+1) n}{8 n^{2}}-\frac{3}{2}-\frac{1}{n}\right]=4^{n} \frac{n-3}{8 n} \geqslant 0 .
\end{aligned}
$$

Donc si $[n, p] \neq[2,2]$ l'hypothèse $k \geqslant p / n$ mène à l'inégalité (17), incompatible avec l'équation

$$
p^{2 n}+p^{2 n-1}+\ldots+1=\left(p^{2}+k\right)^{n}+\left(p^{2}+k\right)^{n-1}+\ldots+1 .
$$

$\mathrm{Au}$ cas: $[n, p]=[2,2]$ correspond la solution $[31,4,2,2,5] \mathrm{de}$ l'équation (1). Done le lemme est entièrement démontré. Alors, de la seconde inégalité de (16): $p>n k$ il s'ensuit que

$$
p-1 \geqslant n k
$$


puisque $k, n, p$ sont des nombres entiers; ainsi les conditions (16) peuvent être mises aussi sous la forme:

$$
1 \leqslant k \leqslant \frac{p-1}{n}
$$

Cela établi, nous pouvons procéder à la démonstration du théorème II. Dans ce but nous allons examiner la variation de la fonction

ou

$$
\begin{aligned}
f(x)=\left(p^{2}+x\right)\left[p^{2 n}+\right. & p^{2 n-1}+\ldots+ \\
& \left.+p+1-\left(p^{2}+x\right)^{n}\right]-\left(p^{2 n}+p^{2 n-1}+\ldots+p^{2}+p\right),
\end{aligned}
$$

$$
f(x)=p^{2 n+2}+p^{2 n+1}+\left(p^{2 n}+p^{2 n-1}+\ldots+p+1\right) x-p-\left(p^{2}+x\right)^{n+1},
$$

$p$ désignant un paramètre $(\geqslant 2)$ qui ne prend que des valeurs entières et absolument indépendantes des valeurs de la variable $x$. Nous allons démontrer que lorsque $x$ varie dans l'intervalle fermé $[0,(p-1) / n]$, la fonction $f(x)$ décroît, en restant toujours positive.

En effet, de (18) nous trouvons en dérivant successivement deux fois par rapport à $x$

$$
\begin{aligned}
f^{\prime}(x) & =p_{: 1}^{2 n}+p^{2 n-1}+\ldots+p+1-(n+1)\left(p^{2}+x\right)^{n} \\
f^{\prime \prime}(x) & =-n(n+1)\left(p^{2}+x\right)^{n-1}<0 \\
& (\text { pour } x \geqslant 0 \text { et pour tout } p \neq 0 \text { réel) } ;
\end{aligned}
$$

et, par conséquent, la fonction $f^{\prime}(x)$ est strictement décroissante pour $x \geqslant 0$. De là nous tirons la conclusion que $f^{\prime}(x)$ - en particulier - est décroissante pourvu que $x \geqslant 0$ et que $p$ soit un certain nombre entier ( $\geqslant 2$ ).

D'autre part, de (19) nous tirons:

$$
\begin{aligned}
f^{\prime}(0) & =p^{2 n-1}+p^{2 n-2}+\ldots+p+1-n p^{2 n} \\
& =\frac{p^{2 n-1}}{p-1}-n p^{2 n} \leqslant p^{2 n}-1-n p^{2 n}<0
\end{aligned}
$$

pour tout $p \geqslant 2$ entier (et pour $n \geqslant 2$ ).

Mais du fait que $f^{\prime}(0)<0$ et que $f^{\prime}(x)$ est une fonction continue et décroissante de $x$ dans l'intervalle $0 \leqslant x<+\infty$ (à $p \geqslant 2$ ) il s'ensuit de manière évidente que $f^{\prime}(x)$ est négative dans le même intervalle, ou que $f(x)$ est une fonction strictement décroissante de $x(p \geqslant 2 ; n \geqslant 2)$ dans l'intervalle: $0 \leqslant x<+\infty$.

En particulier $f(x)$ est une fonction décroissante de $x$ aussi dans l'intervalle

$$
0 \leqslant x \leqslant \frac{p-1}{n}
$$


D'autre part, pour les points extrêmes de l'intervalle nous avons

$$
\begin{aligned}
f\left(\frac{p-1}{n}\right) & =p^{2 n+2}+p^{2 n+1}+\frac{p-1}{n} \cdot \frac{p^{2 n+1}-1}{p-1}-p-\left(p^{2}+\frac{p-1}{n}\right)^{n+1} \\
& =p^{2 n+2}+\left(1+\frac{1}{n}\right) p^{2 n+1}-\left(\frac{1}{n}+p\right)-\left(p^{2}+\frac{p-1}{n}\right)^{n+1} \\
& =-\left(\frac{1}{n}+p\right)+\frac{n+1}{n} p^{2 n}- \\
-\left[\begin{array}{c}
\frac{n+1}{2} \\
n^{2}
\end{array}\right) & \left.p^{2 n-2}(p-1)^{2}+\frac{\left(\begin{array}{c}
n+1 \\
3
\end{array}\right)}{n^{3}} p^{2 n-4}(p-1)^{3}+\ldots+\frac{\left(\begin{array}{l}
n+1 \\
n+1
\end{array}\right)}{n^{n+1}}(p-1)^{n+1}\right] \\
> & -\left(\frac{1}{n}+p\right)+\frac{n+1}{n} p^{2 n}- \\
& -\left[\frac{\left(\begin{array}{c}
n+1 \\
2
\end{array}\right)}{n^{2}} p^{2 n}+\frac{\left(\begin{array}{c}
n+1 \\
3
\end{array}\right)}{n^{3}} p^{2 n-1}+\ldots+\frac{\left(\begin{array}{l}
n+1 \\
n+1
\end{array}\right)}{n^{n+1}} p^{n+1}\right] .
\end{aligned}
$$

Ensuite, pour les valeurs en question de $p$ et $n$, nous trouvons

$$
\begin{aligned}
f\left(\frac{p-1}{n}\right)> & -\left(\frac{1}{n}+p\right)+\frac{n^{\prime}+1}{2 n} p^{2 n}- \\
& -\left[\frac{\left(\begin{array}{c}
n+1 \\
3
\end{array}\right)}{n^{3}} p^{2 n-1}+\frac{\left(\begin{array}{c}
n+1 \\
4
\end{array}\right)}{n^{4}} p^{2 n-2}+\ldots+\frac{\left(\begin{array}{c}
n+1 \\
n+1
\end{array}\right)}{n^{n+1}} p^{n+1}\right] \\
> & -\left(\frac{1}{n}+p\right)+\frac{n+1}{2 n} p^{2 n}- \\
& +\frac{(n+1) p^{n+1}}{6 n}\left[\frac{\left(\begin{array}{c}
n-1 \\
1
\end{array}\right)}{n} p^{n-2}+\frac{\left(\begin{array}{c}
n-1 \\
2
\end{array}\right)}{n^{2}} p^{n-8}+\ldots+\frac{\left(\begin{array}{c}
n-1 \\
n-1
\end{array}\right)}{n^{n-1}}\right] \\
> & -\left(\frac{1}{n}+p\right)+\frac{n+1}{2 n} p^{2 n}-\frac{(n+1) p^{n+1}}{6 n}\left[\left(p+\frac{1}{n}\right)^{n-1}-p^{n-1}\right] \\
= & -\left(\frac{1}{n}+p\right)+\frac{2(n+1) p^{2 n}}{3 n}-\frac{(n+1)}{6 n}\left(p+\frac{1}{n}\right)^{n-1} p^{n+1} .
\end{aligned}
$$

Ainsi nous avons démontré l'inégalité:

$$
f\left(\frac{p-1}{n}\right)>-\left(\frac{1}{n}+p\right)+\frac{2(n+1) p^{2 n}}{3 n}-\frac{(n+1)}{6 n}\left(p+\frac{1}{n}\right)^{n-1} .
$$


Mais, en posant $p \geqslant 2$ et $n \geqslant 2$, nous avons évidemment

c'est-à-dire

$$
\begin{aligned}
\ln \left(1+\frac{1}{p n}\right) & =\frac{1}{p n}-\frac{1}{2 p^{2} n^{2}}+\frac{1}{3 p^{3} n^{3}}-\cdots \\
& <\frac{1}{p(n-1)} \leqslant \frac{1}{2(n-1)}<\frac{\ln 2}{n-1}
\end{aligned}
$$

$$
\ln \left(1+\frac{1}{p n}\right)<\ln 2^{1 /(n-1)}
$$

ou

$$
1+\frac{1}{p n}<2^{1 /(n-1)}, \quad\left(p+\frac{1}{n}\right)^{n-1}<2 p^{n-1} .
$$

De la dernière inégalité et de (21) nous déduisons immédiatement que

$$
\begin{aligned}
f\left(\frac{p-1}{n}\right) & >-\left(\frac{1}{n}+p\right)+\frac{2(n+1)}{3 n} p^{2 n}-\frac{n+1}{3 n} p^{2 n} \\
& =-\left(\frac{1}{n}+p\right)+\frac{n+1}{3 n} p^{2 n}
\end{aligned}
$$

Or, il est évident que pour $p \geqslant 2$ et $n \geqslant 2$ nous avons encore

$$
\frac{n+1}{3 n} p^{2 n}>\frac{p^{2 n}}{3}>2 p>p+\frac{1}{n} \text {. }
$$

Par conséquent

$$
f\left(\frac{p-1}{n}\right)>0 \quad(p \geqslant 2 ; n \geqslant 2) .
$$

Des inégalités (20) et (22) et du fait que la fonction $f(x)$ est continue et décroissante dans l'intervalle

$$
0 \leqslant x \leqslant \frac{p-1}{n}
$$

il s'ensuit que pour chaque valeur de $x$ dans cette intervalle nous aurons

c'est-à-dire

$$
f(x)>0,
$$

$$
\left(p^{2}+x\right)\left[p^{2 n}+p^{2 n-1}+\ldots+p+1-\left(p^{2}+x\right)^{n}\right]-\left(p^{2 n}+p^{2 n-1}+\ldots+p\right)>0
$$

(pour $0 \leqslant x \leqslant(p-1) / n ; p \geqslant 2, n \geqslant 2$ ).

De l'inégalité (23) découle - en particulier - (pour $x=k, k$ - entier et pour $k \leqslant(p-1) / n)$ l'inégalité suivante

$$
\left(p^{2}+k\right)\left[p^{2 n}+p^{2 n-1}+\ldots+p+1-\left(p^{2}+k\right)^{n}\right]>p^{2 n}+p^{2 n-1}+\ldots+p .
$$


Cette dernière inégalité montre que l'égalité (15) n'est pas possible pour

$$
1 \leqslant k \leqslant \frac{p-1}{n} .
$$

Mais comme les inégalités (25) coïncident complètement avec les conditions (16') nous pouvons bien affirmer que l'équation (15) ne possède point de solutions $[p, k]$ soumises aux conditions $\left(16^{\prime}\right)$. De là et du lemme nous tirons la conclusion que l'équation (1) n'a pas de solution $[N, m, n, x, y]$ pour $m=2 n$ sauf $[31,4,2,2,5]$. Le théorème II se trouve ainsi démontré.

Remarque. A l'aide du théorème $I$ et de théorèmes analogues au théorème II on peut traiter d'autres cas de l'équation (1); par exemple: $m=3 n, m=4 n$, etc.

Enfin, je tiens, à remercier à M. A. Schinzel dont les remarques ont permis d'abréger la démonstration du lemme.

\section{Travaux cités}

[1] R. Goormaghtigh, I. M., 1917-88, 153.

[2] H. J. Kanold, Über einen Satz von L. E. Dickson, Math. Ann. (1956), p. 246.

[3] A. Schinzel et A. Mąkowski, Sur l'équation indéterminée de R. Goormaghtigh, Mathesis 58 (1959), pp. 128.141. 